\title{
On the strict positivity of entropy production
}

\author{
Vojkan Jakšić $^{1}$ and Claude-Alain Pillet ${ }^{2}$ \\ ${ }^{1}$ Department of Mathematics and Statistics \\ McGill University \\ 805 Sherbrooke Street West \\ Montreal, QC, H3A 2K6, Canada \\ ${ }^{2}$ FRUMAM \\ CPT-CNRS, UMR 6207 \\ Université du Sud, Toulon-Var, B.P. 20132 \\ F-83957 La Garde Cedex, France
}

December 22, 2006

\begin{abstract}
We refine an abstract result of the authors [JP3] concerning the strict positivity of entropy production of $L^{1}$-asymptotically Abelian $C^{*}$-dynamical systems and apply the new result to a model describing finitely many free Fermi gas reservoirs coupled by local interactions. In particular, assuming that the reservoirs are initially not at equal temperatures or chemical potentials, we show that there is a dense set of local interactions such that the entropy production of the system is strictly positive for sufficiently small non-zero coupling.
\end{abstract}




\section{Introduction}

For notational purposes we start with a brief review of certain aspects of algebraic nonequilibrium quantum statistical mechanics. Our terminology is standard [BR1, BR2] and we shall assume that the reader is familiar with results described in the review [JP3] and in the recent lecture notes [AJPP].

Our starting point is a $C^{*}$-dynamical system $\left(\mathcal{O}, \tau_{0}\right)$ with $\tau_{0}^{t}=\mathrm{e}^{t \delta_{0}}$. To avoid discussion of trivial cases, we shall always assume that $\tau_{0}$ is a not a trivial dynamics, i.e., that $\delta_{0} \neq 0$. For any $\mathcal{S} \subset \mathcal{O}$ we denote $\mathcal{S}_{\text {self }}=\left\{A \in \mathcal{S} \mid A=A^{*}\right\}$. For $V \in \mathcal{O}_{\text {self }}$ and $\lambda \in \mathbb{R}$ set $\delta_{\lambda V}=\delta_{0}+\mathrm{i} \lambda[V, \cdot]$ and denote by $\tau_{\lambda V}^{t}=\mathrm{e}^{t \delta_{\lambda V}}$ the perturbed $C^{*}$-dynamics. We shall assume a strong form of ergodicity $\left(L^{1}\right.$-asymptotic Abelianness $)$ of $\left(\mathcal{O}, \tau_{\lambda V}\right)$ in the following form:

(A1) There exists a norm dense $*$-subalgebra $\mathcal{A} \subset \mathcal{O}$ with the property: For any $V \in \mathcal{A}_{\text {self }}$ there exists $\lambda_{V}>0$ such that

$$
\int_{-\infty}^{\infty}\left\|\left[V, \tau_{\lambda V}^{t}(A)\right]\right\| \mathrm{d} t<\infty
$$

holds for all $|\lambda|<\lambda_{V}$ and $A \in \mathcal{A}$.

A well-known consequence of (A1) is that for $V \in \mathcal{A}_{\text {self }}$ and $|\lambda|<\lambda_{V}$ the strong limits

$$
\begin{aligned}
& \gamma_{\lambda V}^{ \pm}=\lim _{t \rightarrow \pm \infty} \tau_{\lambda V}^{-t} \circ \tau_{0}^{t}, \\
& \alpha_{\lambda V}^{ \pm}=\lim _{t \rightarrow \pm \infty} \tau_{0}^{-t} \circ \tau_{\lambda V}^{t},
\end{aligned}
$$

exist and are $*$-automorphisms of $\mathcal{O}$. Moreover, $\left(\gamma_{\lambda V}^{ \pm}\right)^{-1}=\alpha_{\lambda V}^{ \pm}$.

Let $\omega$ be a given $\tau_{0}$-invariant reference state on $\mathcal{O}$. Let $V \in \mathcal{A}_{\text {self }}$ and $|\lambda|<\lambda_{V}$. Then for all $A \in \mathcal{O}$,

$$
\lim _{t \rightarrow \pm \infty} \omega\left(\tau_{\lambda V}^{t}(A)\right)=\omega_{\lambda V}^{ \pm}(A)
$$

where $\omega_{\lambda V}^{ \pm}=\omega \circ \alpha_{\lambda V}^{ \pm}$. The states $\omega_{\lambda V}^{ \pm}$are $\tau_{\lambda V}$-invariant and $\omega_{\lambda V}^{+}$is sometimes called non-equilibrium steady state (NESS) of the locally perturbed quantum dynamical system $\left(\mathcal{O}, \tau_{\lambda V}, \omega\right)$ [Ru1].

Concerning $\omega$, we shall assume the following:

(A2) For all $A_{1}, A_{2}, A_{3} \in \mathcal{O}$,

$$
\lim _{\min _{i \neq j}\left|t_{i}-t_{j}\right| \rightarrow \infty} \omega\left(\tau_{0}^{t_{1}}\left(A_{1}\right) \tau_{0}^{t_{2}}\left(A_{2}\right) \tau_{0}^{t_{3}}\left(A_{3}\right)\right)=\omega\left(A_{1}\right) \omega\left(A_{2}\right) \omega\left(A_{3}\right) .
$$

(A3) There exists a $C^{*}$-dynamics $\varsigma$ on $\mathcal{O}$ such that $\omega$ is a $(\varsigma,-1)$-KMS state. Moreover, $\mathcal{A} \subset \operatorname{Dom}\left(\delta_{\varsigma}\right)$, where $\delta_{\varsigma}$ is the generator of $\varsigma$. We shall assume that $\varsigma$ is non-trivial, i.e., that $\delta_{\varsigma} \neq 0$. 
Assumption (A2) and the first part of (A3) imply that the system $\left(\mathcal{O}, \tau_{0}, \omega\right)$ has the property of return to equilibrium: For all $\omega$-normal states $\eta$ and $A \in \mathcal{O}$,

$$
\lim _{|t| \rightarrow \infty} \eta\left(\tau_{0}^{t}(A)\right)=\omega(A)
$$

see $[\mathrm{JP}]$. The second part of (A3) ensures that the entropy production observable

$$
\sigma_{\lambda V}=\lambda \delta_{\varsigma}(V)
$$

is well defined for all $V \in \mathcal{A}_{\text {self }}$. The entropy production of the NESS $\omega_{\lambda V}^{+}$is defined by

$$
\operatorname{Ep}\left(\omega_{\lambda V}^{+}\right)=\omega_{\lambda V}^{+}\left(\sigma_{\lambda V}\right)
$$

Denote by $\operatorname{Ent}(\cdot \mid \cdot)$ the relative entropy of Araki with sign and ordering convention of [BR2]. The entropy balance equation

$$
\operatorname{Ent}\left(\omega \circ \tau_{\lambda V}^{t} \mid \omega\right)=-\int_{0}^{t} \omega\left(\tau_{\lambda V}^{s}\left(\sigma_{\lambda V}\right)\right) \mathrm{d} s
$$

holds [JP2]. This relation implies

$$
\operatorname{Ep}\left(\omega_{\lambda V}^{+}\right) \geq 0
$$

$[\mathrm{Ru} 2, \mathrm{JP} 2]$. From the point of view of non-equilibrium statistical mechanics, the perturbed system is thermodynamically non-trivial iff the entropy production of its NESS is strictly positive.

Suppose that the unperturbed system is initially in thermal equilibrium, namely that $\omega$ is a $\left(\tau_{0}, \beta\right)$-KMS state on $\mathcal{O}$ for some $\beta \in \mathbb{R} \cup\{ \pm \infty\}$. If $\beta \in \mathbb{R} \backslash\{0\}$, then $\delta_{\varsigma}=-\beta \delta_{0}, \sigma_{\lambda V}=$ $\delta_{\lambda V}(-\beta \lambda V)$ and since $\omega_{\lambda V}^{+}$is $\tau_{\lambda V}$-invariant, $\operatorname{Ep}\left(\omega_{\lambda V}^{+}\right)=0$ for all $V \in \mathcal{A}_{\text {self }}$ and $|\lambda|<\lambda_{V}$. The cases $\beta= \pm \infty, 0$ are not possible under our assumptions. If $\beta= \pm \infty$, then the first part of (A3) and Proposition 5.3.19 in [BR2] imply that $\tau_{0}$ is trivial, i.e., that $\delta_{0}=0$. If $\beta=0$, then the first part of (A3) implies that $\varsigma$ is trivial, i.e., that $\delta_{\varsigma}=0$.

On physical grounds one expects that if $\omega$ is not a thermal equilibrium state, then $\operatorname{Ep}\left(\omega_{\lambda V}^{+}\right)$ is strictly positive for a large class of perturbations $\lambda V$, and this is the question we shall address in this note.

The next assumption concerns the rate of convergence of $\omega\left(\tau_{\lambda V}^{t}\left(\sigma_{\lambda V}\right)\right)$ to its limiting value $\omega_{\lambda V}^{+}\left(\sigma_{\lambda V}\right)$.

(A4) For all $V \in \mathcal{A}_{\text {self }}$,

$$
C_{V}=\sup _{0<|\lambda|<\lambda_{V}, t \geq 0}\left|\frac{1}{\lambda} \int_{0}^{t}\left(\omega\left(\tau_{\lambda V}^{s}\left(\sigma_{\lambda V}\right)\right)-\omega_{\lambda V}^{+}\left(\sigma_{\lambda V}\right)\right) \mathrm{d} s\right|<\infty .
$$

Theorem 1.1 Suppose that (A1)-(A4) hold and that $\omega$ is not a $\left(\tau_{0}, \beta\right)-K M S$ state for some $\beta \in \mathbb{R} \backslash\{0\}$. Then there exists a set $\mathcal{V} \subset \mathcal{A}_{\text {self }}$ such that: 
1. $\mathcal{V}$ is norm-dense in $\mathcal{A}_{\text {self }}$ (and hence in $\mathcal{O}_{\text {self }}$ ).

2. For all $V \in \mathcal{V}$ there exists $\Lambda_{V}>0$ such that

$$
\operatorname{Ep}\left(\omega_{\lambda V}^{+}\right)>0
$$

for $0<|\lambda|<\Lambda_{V}$

The proof of Theorem 1.1 is given in Section 2 and is based on the results and heuristics described in [JP3] (see in particular Theorem 4.7 in this reference).

In the rest of this introduction we shall describe an application of Theorem 1.1 to statistical mechanics of open Fermi systems. We shall consider the same model as in [JOP]. All our results and proofs will heavily rely on the results of [JOP].

A free Fermi gas is described by the $C^{*}$-dynamical system $\left(\mathfrak{O}, \tau_{0}\right)$ where:

(i) $\mathfrak{O}=\operatorname{CAR}(\mathfrak{h})$ is the CAR algebra over the single particle Hilbert space $\mathfrak{h}$.

(ii) $\tau_{0}^{t}$ is the group of Bogoliubov $*$-automorphisms generated by the single particle Hamiltonian $h_{0}$,

$$
\tau_{0}^{t}\left(a^{\#}(f)\right)=a^{\#}\left(\mathrm{e}^{\mathrm{i} t h_{0}} f\right)
$$

where $a^{*}(f) / a(f)$ are the creation/annihilation operators associated to $f \in \mathfrak{h}$ and $a^{\#}$ stands for either $a$ or $a^{*}$.

Let $\mathcal{O}$ be the $\tau_{0}$-invariant $C^{*}$-subalgebra of $\mathfrak{O}$ generated by $\left\{a^{*}(f) a(g) \mid f, g \in \mathfrak{h}\right\}$ and $\mathbb{1}$. Physical observables are gauge invariant and hence elements of $\mathcal{O}$.

We make the assumption:

(B1) There exist a dense vector subspace $\mathcal{D} \subset \mathfrak{h}$ and $\rho \geq 0$ such that the functions

$$
\mathbb{R} \ni t \mapsto|t|^{\rho}\left(f, \mathrm{e}^{\mathrm{i} t h_{0}} g\right),
$$

are in $L^{1}(\mathbb{R}, \mathrm{d} t)$ for all $f, g \in \mathcal{D}$.

This assumption implies that $h_{0}$ has purely absolutely continuous spectrum.

Let $\mathcal{A} \subset \mathcal{O}$ be the collection of the elements of the form

$$
A=\sum_{k=1}^{K} \prod_{j=1}^{n_{k}} a^{*}\left(f_{k j}\right) a\left(g_{k j}\right)+c \mathbb{1},
$$

where $K, n_{1}, \ldots, n_{K}$ are finite with $\bar{n} \equiv \max _{k} n_{k} \geq 2, c \in \mathbb{C}$, and

$$
\mathcal{F}(A) \equiv\left\{f_{k j}, g_{k j} \mid k=1, \ldots, K ; j=1, \ldots, n_{k}\right\} \subset \mathcal{D} .
$$


To indicate the dependence of $K$ and $\bar{n}$ on $A$ we will denote them by $K_{A}$ and $\bar{n}_{A}$. It is easy to see that $\mathcal{A}$ is a $*$-subalgebra of $\mathcal{O}$ containing all the elements of the form $a^{*}(f) a(g)$ with $f, g \in \mathcal{D}$ as well as $\mathbb{1}$. Since $\mathcal{D}$ is dense in $\mathfrak{h}$ it follows that $\mathcal{A}$ is a norm dense $*$-subalgebra of $\mathcal{O}$.

To each pair $A, B \in \mathcal{A}$ we associate the constant

$$
\ell_{A \mid B}=\int_{-\infty}^{\infty} \max _{f \in \mathcal{F}(A), g \in \mathcal{F}(B)}\left|\left(f, \mathrm{e}^{\mathrm{i} t h_{0}} g\right)\right| \mathrm{d} t .
$$

For $A \in \mathcal{A}$ we write $\ell_{A}=\ell_{A \mid A}$ and set

$$
\begin{aligned}
M_{A} & =K_{A}(\max \{1,\|f\| \mid f \in \mathcal{F}(A)\})^{2 \bar{n}_{A}-1}, \\
\lambda_{A} & =\left(2 \bar{n}_{A} M_{A} \ell_{A}\right)^{-1} \frac{\left(2 \bar{n}_{A}-2\right)^{2 \bar{n}_{A}-2}}{\left(2 \bar{n}_{A}-1\right)^{2 \bar{n}_{A}-1}} .
\end{aligned}
$$

The following result was proven in [JOP] (see also [Ro, BM1, AM, BM2, FMU]):

Theorem 1.2 Suppose that (B1) holds. Then:

1. For all $V \in \mathcal{A}_{\text {self }}$ and $A \in \mathcal{A}$,

$$
\sup _{|\lambda| \leq \lambda_{V}} \int_{-\infty}^{\infty}\left\|\left[V, \tau_{\lambda V}^{t}(A)\right]\right\| \mathrm{d} t \leq \frac{2 M_{A}}{\lambda_{V}}\left[\left(1+\frac{\ell_{V \mid A}}{\left(2 \bar{n}_{V}-2\right) \ell_{V}}\right)^{2 \bar{n}_{A}}-1\right] .
$$

In particular, Assumption (Al) holds.

2. For all $V \in \mathcal{A}_{\text {self }}$ and $A \in \mathcal{A}$ the functions

$$
\lambda \mapsto \gamma_{\lambda V}^{ \pm}(A) \in \mathcal{O}, \quad \lambda \mapsto \alpha_{\lambda V}^{ \pm}(A) \in \mathcal{O},
$$

are analytic for $|\lambda|<\lambda_{V}$.

Let $T$ be a self-adjoint operator on $\mathfrak{h}$ satisfying $0 \leq T \leq 1$ and $\left[T, \mathrm{e}^{\mathrm{i} t h_{0}}\right]=0$ for all $t$. In what follows $\omega$ is the gauge invariant quasi-free state on $\mathfrak{O}$ generated by $T$. The state $\omega$ is $\tau_{0}$-invariant and is a $\left(\tau_{0}, \beta\right)$-KMS state on $\mathfrak{O}$ for some $\beta \in \mathbb{R} \backslash\{0\}$ if and only if

$$
T=\frac{1}{1+\mathrm{e}^{\beta h_{0}}} \text {. }
$$

Assumption (B1) implies that for all $A_{1}, \cdots, A_{n} \in \mathfrak{O}$,

$$
\lim _{\min _{i \neq j}\left|t_{i}-t_{j}\right| \rightarrow \infty} \omega\left(\tau_{0}^{t_{1}}\left(A_{1}\right) \cdots \tau_{0}^{t_{n}}\left(A_{n}\right)\right)=\omega\left(A_{1}\right) \cdots \omega\left(A_{n}\right),
$$

and so (A2) holds. Assumption (B1) also implies that $\omega \uparrow \mathcal{O}$ is a $\left(\tau_{0}, \beta\right)$-KMS state on $\mathcal{O}$ for some $\beta \in \mathbb{R} \backslash\{0\}$ if and only if

$$
T=\frac{1}{1+\mathrm{e}^{\beta\left(h_{0}-\mu\right)}},
$$

for some $\mu \in \mathbb{R}$.

Our final assumption is 
(B2) $\operatorname{Ker} T=\operatorname{Ker}(1-T)=\{0\}$ and $T \neq 1 / 2$. Moreover, if $h_{T}=\log T(1-T)^{-1}$, then $\mathcal{D} \subset \operatorname{Dom}\left(h_{T}\right)$ and $h_{T} \mathcal{D} \subset \mathcal{D}$.

If $\varsigma$ is the group of Bogoliubov $*$-automorphisms on $\mathfrak{O}$ generated by $h_{T}$, then $\omega$ is the unique $(\varsigma,-1)$-KMS state on $\mathfrak{O}$ and a $(\varsigma,-1)$-KMS state on $\mathcal{O}$. Since $T \neq 1 / 2, \varsigma$ is non-trivial, and so (B2) implies (A3). In particular, the entropy production observable $\sigma_{\lambda V}$ is well-defined for all $V \in \mathcal{A}_{\text {self }}$ and $\lambda \in \mathbb{R}$, and $\sigma_{\lambda V} \in \mathcal{A}_{\text {self }}$.

Theorem 1.3 Suppose that (B1) and (B2) hold for some $\rho>1$ and that $\omega$ is not a $\left(\tau_{0}, \beta\right)-K M S$ state on $\mathcal{O}$ for any $\beta \in \mathbb{R} \backslash\{0\}$. Then there exists a set $\mathcal{V} \subset \mathcal{A}_{\text {self }}$ such that:

1. $\mathcal{V}$ is norm-dense in $\mathcal{A}_{\text {self }}$ (and hence in $\mathcal{O}_{\text {self }}$ ).

2. For all $V \in \mathcal{V}$ and $0<|\lambda|<\lambda_{V}$,

$$
\operatorname{Ep}\left(\omega_{\lambda V}^{+}\right)>0
$$

except possibly for a discrete set of $\lambda$ 's which can accumulate only at $\left\{ \pm \lambda_{V}\right\}$.

3. For all $V \in \mathcal{V}$ and $W \in \mathcal{A}_{\text {self }}$ there exists $\epsilon_{V W}>0$ such that $V+\epsilon W \in \mathcal{V}$ for all $|\epsilon|<\epsilon_{V W}$.

This theorem can be applied to the open quantum systems studied in [JOP]. Suppose that for some $M \geq 2$,

$$
\mathfrak{h}=\bigoplus_{j=1}^{M} \mathfrak{h}_{j}, \quad h_{0}=\bigoplus_{j=1}^{M} h_{j}, \quad T=\bigoplus_{j=1}^{M} \frac{1}{1+\mathrm{e}^{\beta_{j}\left(h_{j}-\mu_{j}\right)}},
$$

where $h_{j}$ 's are self-adjoint operators on the Hilbert subspaces $\mathfrak{h}_{j}, \beta_{j} \in \mathbb{R} \backslash\{0\}$, and $\mu_{j} \in \mathbb{R}$. We denote by $p_{j}$ the orthogonal projections onto $\mathfrak{h}_{j}$. The subalgebras $\mathfrak{O}_{j}=\operatorname{CAR}\left(\mathfrak{h}_{j}\right)$ describe reservoirs $\mathcal{R}_{j}$ which are initially in equilibrium at inverse temperatures $\beta_{j}$ and chemical potentials $\mu_{j}$. The perturbation $\lambda V$ describes the interaction between the reservoirs (and, possibly, self-interactions within the reservoirs) and allows for the flow of heat and charges within the system. Clearly,

$$
h_{T}=-\bigoplus_{j=1}^{M} \beta_{j}\left(h_{j}-\mu_{j}\right)
$$

and

$$
\sigma_{\lambda V}=-\sum_{j=1}^{M} \beta_{j}\left(\Phi_{j}-\mu_{j} \mathcal{J}_{j}\right)
$$


where

$$
\begin{aligned}
& \Phi_{j}=\lambda \sum_{k=1}^{K} \sum_{l=1}^{n_{k}}\left(\prod_{i=1}^{l-1} a^{*}\left(f_{k i}\right) a\left(g_{k i}\right)\right) \begin{array}{r}
\left\{a^{*}\left(\mathrm{i} h_{j} p_{j} f_{k l}\right) a\left(g_{k l}\right)\right. \\
\left.+a^{*}\left(f_{k l}\right) a\left(\mathrm{i} h_{j} p_{j} g_{k l}\right)\right\}
\end{array}\left(\prod_{i=l+1}^{n_{k}} a^{*}\left(f_{k i}\right) a\left(g_{k i}\right)\right), \\
& \mathcal{J}_{j}=\lambda \sum_{k=1}^{K} \sum_{l=1}^{n_{k}}\left(\prod_{i=1}^{l-1} a^{*}\left(f_{k i}\right) a\left(g_{k i}\right)\right) \begin{array}{l}
\left\{a^{*}\left(\mathrm{i} p_{j} f_{k l}\right) a\left(g_{k l}\right)\right. \\
\left.+a^{*}\left(f_{k l}\right) a\left(\mathrm{i} p_{j} g_{k l}\right)\right\}
\end{array}\left(\prod_{i=l+1}^{n_{k}} a^{*}\left(f_{k i}\right) a\left(g_{k i}\right)\right) .
\end{aligned}
$$

The observable $\Phi_{j} / \mathcal{J}_{j}$ describe the heat/charge flux out of the reservoir $\mathcal{R}_{j}$. The conservation laws

$$
\sum_{j=1}^{M} \omega_{\lambda V}^{+}\left(\Phi_{j}\right)=0, \quad \sum_{j=1}^{M} \omega_{\lambda V}^{+}\left(\mathcal{J}_{j}\right)=0
$$

hold, and the strict positivity of the entropy production implies that the NESS $\omega_{\lambda V}^{+}$carries nonvanishing fluxes.

A consequence of Theorem 1.3 is:

Corollary 1.4 Suppose that $h_{0}$ and $T$ have the form (1.3) and that either the $\beta_{j}$ 's are not all equal or the $\beta_{j} \mu_{j}$ 's are not all equal. Suppose also that (B1) holds for some $\rho>1$ and that $p_{j} \mathcal{D} \subset \mathcal{D}$ and $h_{j} p_{j} \mathcal{D} \subset \mathcal{D}$ for all $j$. Then all conclusions of Theorem 1.3 hold.

Remark. For specific interactions $V$ one can compute $\omega_{\lambda V}^{+}\left(\sigma_{\lambda V}\right)$ to the first non-trivial order in $\lambda$ and hence establish the strict positivity of entropy production by a perturbative calculation [FMU, JP4, AS]. Theorem 1.3 and Corollary 1.4 complement such results by establishing the strict positivity of the entropy production for a generic perturbation $\lambda V$.

We finish with some concrete examples to which Corollary 1.4 applies. Let $\mathbb{Z}_{+}^{d}=\mathbb{Z}_{+} \times$ $\mathbb{Z}^{d-1}$, where $\mathbb{Z}_{+}=\{0,1, \cdots\}$. If $\mathfrak{h}_{j}=\ell^{2}\left(\mathbb{Z}^{d_{j}}\right)$ or $\mathfrak{h}_{j}=\ell\left(\mathbb{Z}_{+}^{d_{j}}\right)$ and $h_{j}$ is the usual discrete Laplacian on these spaces, then one can always find $\mathcal{D}$ so that Corollary 1.4 holds. One can take $\mathcal{D}$ to be the vector space of compactly supported functions if $d_{j} \geq 5$ and $\mathfrak{h}_{j}=\ell^{2}\left(\mathbb{Z}^{d_{j}}\right)$ or $d_{j} \geq 2$ and $\mathfrak{h}=\ell\left(\mathbb{Z}_{+}^{d_{j}}\right)$. In the continuous case, if $\mathfrak{h}_{j}=L^{2}\left(\mathbb{R}^{d_{j}}\right)$ or $\mathfrak{h}_{j}=L^{2}\left(\mathbb{R}_{+}^{d_{j}}\right)$ and $h_{j}=-\Delta$ (with Dirichlet boundary condition in the case of $\mathbb{R}_{+}^{d_{j}}$ ), again one can always find $\mathcal{D}$ so that Corollary 1.4 holds. One can take $\mathcal{D}$ to be the vector space of $C^{\infty}$ compactly supported functions if $\mathfrak{h}_{j}=L^{2}\left(\mathbb{R}^{d_{j}}\right)$ and $d_{j} \geq 5$ or $\mathfrak{h}_{j}=L^{2}\left(\mathbb{R}_{+}^{d_{j}}\right)$ and $d_{j} \geq 2$.

Acknowledgments. The research of V.J. was partly supported by NSERC and part of this work was done during his visit to CPT-CNRS. 


\section{Proof of Theorem 1.1}

Throughout the proof we shall freely use the well known properties of the relative entropy functional discussed in Section II.4 of [OP]. We denote by $\mathcal{N}_{\omega}$ the set of all $\omega$-normal states on $\mathcal{O}$.

Proposition 2.1 Let $V \in \mathcal{A}_{\text {self }}$ and $0<|\lambda|<\lambda_{V}$ be such that $\operatorname{Ep}\left(\omega_{\lambda V}^{+}\right)=0$. Then the following hold:

(i) $\omega_{\lambda V}^{+} \in \mathcal{N}_{\omega}$.

(ii) $\omega_{\lambda V}^{-}=\omega_{\lambda V}^{+}$

Proof. Since $V$ and $\lambda$ are fixed, we shall not use the subscripts $\lambda V$ throughout the proof. Hence $\tau=\tau_{\lambda V}, \omega^{ \pm}=\omega_{\lambda V}^{ \pm}$, etc.

As a consequence of Assumption (A4) and of the entropy balance equation (1.1) the condition $\operatorname{Ep}\left(\omega^{+}\right)=0$ implies the lower bound

$$
-\infty<-C_{V}|\lambda| \leq \operatorname{Ent}\left(\omega \circ \tau^{t} \mid \omega\right) \leq 0
$$

for $t \geq 0$. It follows that the set of states $\left\{\omega \circ \tau^{t} \mid t \geq 0\right\}$ is weak*-precompact in $\mathcal{N}_{\omega}$ and the limiting state $\omega^{+}$is $\omega$-normal. This proves (i). Relation

$$
\operatorname{Ent}\left(\omega \circ \tau^{t} \mid \omega\right)=\operatorname{Ent}\left(\omega \mid \omega \circ \tau^{-t}\right)
$$

and the weak* upper semi-continuity of the relative entropy yields

$$
\operatorname{Ent}\left(\omega \mid \omega^{-}\right) \geq \limsup _{t \rightarrow+\infty} \operatorname{Ent}\left(\omega \mid \omega \circ \tau^{-t}\right) \geq \inf _{t \geq 0} \operatorname{Ent}\left(\omega \circ \tau^{t} \mid \omega\right)>-\infty .
$$

Thus $\omega$ is $\omega^{-}$-normal and hence $\mathcal{N}_{\omega} \subset \mathcal{N}_{\omega^{-}}$(see e.g. Theorem 2.30 in [P]). Since the dynamical systems systems $\left(\mathcal{O}, \tau_{0}, \omega\right)$ and $\left(\mathcal{O}, \tau, \omega^{-}\right)$are isomorphic, $\left(\mathcal{O}, \tau, \omega^{-}\right)$also has the property of return to equilibrium: For all $\eta \in \mathcal{N}_{\omega^{-}}$and $A \in \mathcal{O}$,

$$
\lim _{|t| \rightarrow+\infty} \eta\left(\tau^{t}(A)\right)=\omega^{-}(A) .
$$

Taking $\eta=\omega^{+}$we deduce (ii).

Proposition 2.2 Let $V \in \mathcal{A}_{\text {self }}$ be such that for some sequence $\lambda_{n} \rightarrow 0, \lambda_{n} \neq 0$,

$$
\operatorname{Ep}\left(\omega_{\lambda_{n} V}^{+}\right)=0 .
$$

Then

$$
\int_{-\infty}^{\infty} \omega\left(\left[V, \tau_{0}^{t}(A)\right]\right) \mathrm{d} t=0,
$$

for all $A \in \mathcal{A}$. 
Proof. Since for $|\lambda|<\lambda_{V}$ and $A \in \mathcal{A}$

$$
\omega(A)=\omega_{\lambda V}^{ \pm}\left(\gamma_{\lambda V}^{ \pm}(A)\right)=\lim _{t \rightarrow \pm \infty} \omega_{\lambda V}^{ \pm}\left(\tau_{0}^{t}(A)\right)
$$

we have

$$
\omega(A)-\omega_{\lambda V}^{ \pm}(A)=\mp \mathrm{i} \lambda \int_{0}^{\infty} \omega_{\lambda V}^{ \pm}\left(\left[V, \tau_{0}^{ \pm t}(A)\right]\right) \mathrm{d} t
$$

and hence

$$
\omega_{\lambda V}^{+}(A)-\omega_{\lambda V}^{-}(A)=\mathrm{i} \lambda \int_{-\infty}^{\infty} \omega_{\lambda V}^{s(t)}\left(\left[V, \tau_{0}^{t}(A)\right]\right) \mathrm{d} t,
$$

where $s(t)=\operatorname{sign}(t)$. Taking $\lambda=\lambda_{n}$, we deduce from Proposition 2.1 that

$$
\int_{-\infty}^{\infty} \omega_{\lambda_{n} V}^{+}\left(\left[V, \tau_{0}^{t}(A)\right]\right) \mathrm{d} t=0 .
$$

As in the proof of Proposition 2.1, $\operatorname{Ep}\left(\omega_{\lambda_{n} V}^{+}\right)=0$ implies

$$
-C_{V}\left|\lambda_{n}\right| \leq \operatorname{Ent}\left(\omega \circ \tau_{\lambda_{n} V}^{t} \mid \omega\right) \leq 0
$$

for $t \geq 0$ and the weak* upper semi-continuity of the relative entropy yields

$$
\operatorname{Ent}\left(\omega_{\lambda_{n} V}^{+} \mid \omega\right)=O\left(\left|\lambda_{n}\right|\right)
$$

This estimate and the entropic inequality

$$
\left\|\omega_{\lambda_{n} V}^{+}-\omega\right\|^{2} \leq-2 \operatorname{Ent}\left(\omega_{\lambda_{n} V}^{+} \mid \omega\right)
$$

yield that

$$
\lim _{n \rightarrow \infty}\left\|\omega_{\lambda_{n} V}^{+}-\omega\right\|=0
$$

Relations (2.6), (2.7), Assumption (A1) and the dominated convergence theorem imply the statement. $\square$

Proof of Theorem 1.1 Assume that the statement does not hold. Then there exist $V_{0} \in \mathcal{A}_{\text {self }}$ and $\epsilon>0$ such that for all $W \in \mathcal{A}_{\text {self }}$ satisfying $\left\|V_{0}-W\right\|<\epsilon$ there exists a ( $W$-dependent) sequence $\lambda_{n} \rightarrow 0, \lambda_{n} \neq 0$, such that $\operatorname{Ep}\left(\omega_{\lambda_{n} W}^{+}\right)=0$. Proposition 2.2 yields that

$$
\int_{-\infty}^{\infty} \omega\left(\left[W, \tau_{0}^{t}(A)\right]\right) \mathrm{d} t=0
$$

Let $V \in \mathcal{A}_{\text {self }}$ be given and let $0<s<\epsilon /\|V\|$. Taking $W=V_{0}+s V$ in (2.8), we derive that for all $A \in \mathcal{A}$

$$
\int_{-\infty}^{\infty} \omega\left(\left[V_{0}, \tau_{0}^{t}(A)\right]\right) \mathrm{d} t+s \int_{-\infty}^{\infty} \omega\left(\left[V, \tau_{0}^{t}(A)\right]\right) \mathrm{d} t=0 .
$$


Since the first term in (2.9) is vanishing, we deduce that for all $V \in \mathcal{A}_{\text {self }}$ and $A \in \mathcal{A}$,

$$
\int_{-\infty}^{\infty} \omega\left(\left[V, \tau_{0}^{t}(A)\right]\right) \mathrm{d} t=0
$$

and so for all $A, B \in \mathcal{A}$,

$$
\int_{-\infty}^{\infty} \omega\left(\left[A, \tau_{0}^{t}(B)\right]\right) \mathrm{d} t=0 .
$$

By the well-known result of Bratteli, Kishimoto and Robinson (see [BKR] and Theorem 5.4.20 in [BR2]), (A1), (A2) and (2.10) imply that $\omega$ is a $\left(\tau_{0}, \beta\right)$-KMS state for some $\beta \in \mathbb{R} \cup\{ \pm \infty\}$. Our assumptions exclude the cases $\beta= \pm \infty, 0$, and the result follows.

\section{Proof of Theorem 1.3}

Throughout this subsection $\rho$ is the constant in Assumption (B1). In the first two propositions we assume only that $\rho \geq 0$. Set $\langle t\rangle=\sqrt{1+t^{2}}$,

$$
\ell_{V, \rho}=\int_{0}^{\infty} \max _{f, g \in \mathcal{F}(V)}\langle t\rangle^{\rho}\left|\left(f, \mathrm{e}^{\mathrm{i} t h_{0}} g\right)\right| \mathrm{d} t
$$

and

$$
\lambda_{V, \rho}=\left(2 \bar{n}_{V} M_{V} \ell_{V, \rho}\right)^{-1} \frac{\left(2 \bar{n}_{V}-2\right)^{2 \bar{n}_{V}-2}}{\left(2 \bar{n}_{V}-1\right)^{2 \bar{n}_{V}-1}} .
$$

Proposition 3.1 Suppose that (B1) holds for some $\rho \geq 0$ and let $V \in \mathcal{A}_{\text {self. Then for all }}$ $0<\Lambda<\lambda_{V, \rho}$ and $A \in \mathcal{A}$,

$$
\sup _{|\lambda| \leq \Lambda} \int_{0}^{\infty}\langle t\rangle^{\rho}\left\|\left[V, \tau_{\lambda V}^{t}(A)\right]\right\| \mathrm{d} t<\infty .
$$

Proof. This proposition is a consequence of the arguments used in the proof of Theorem 1.1 in [JOP]. We shall use freely the terminology and estimates of the Section 2.1 in [JOP].

Let $\mathcal{T}_{n}$ be the set of all rooted trees with the nodes 0 (the root), $1, \cdots, n$. A function $T$ : $\{0, \ldots, n\} \rightarrow\{0, \ldots, n\}$ is called a climber of order $n$ if $T(0)=0$ and $T(j)<j$ for all $j \in\{1, \ldots, n\}$. There is one-one correspondence between climbers and rooted trees. We set $r_{0}=\left|T^{-1}(\{0\})\right|-1$ and $r_{j}=\left|T^{-1}(\{j\})\right|$ for $j \in\{1, \ldots, n\}\left(r_{j}\right.$ is the number of childs of the node $j$ ). To $T \in \mathcal{T}_{n}$ we associate the constant

$$
\bar{N}(T)=\frac{\left(2 \bar{n}_{A}\right) !}{\left(2 \bar{n}_{A}-r_{0}\right) !} \prod_{j=1}^{n-1} \frac{\left(2 \bar{n}_{V}\right) !}{\left(2 \bar{n}_{V}-r_{j}-1\right) !}
$$


if $r_{0} \leq 2 \bar{n}_{A}$ and $r_{j} \leq 2 \bar{n}_{V}-1$, otherwise $\bar{N}(T)=0$. Set

$$
G_{k}(t) \equiv M_{V} \begin{cases}\max _{f \in \mathcal{F}(V), g \in \mathcal{F}(A)}\left|\left(f, \mathrm{e}^{\mathrm{i} t h_{0}} g\right)\right| & \text { for } k=0 \\ \max _{f, g \in \mathcal{F}(V)}\left|\left(f, \mathrm{e}^{\mathrm{i} t h_{0}} g\right)\right| & \text { for } k>0 .\end{cases}
$$

In [JOP] the following estimate was established:

$$
\left\|\left[V, \tau_{\lambda V}^{t}(A)\right]\right\| \leq M_{A} \sum_{n=1}^{\infty}|\lambda|^{n-1} \sum_{T \in \mathcal{T}_{n}} \bar{N}(T) \int_{0=s_{n} \leq s_{n-1} \cdots \leq s_{0}=t} \prod_{j=1}^{n} G_{T(j)}\left(s_{T(j)}-s_{j}\right) \mathrm{d} s_{1} \cdots \mathrm{d} s_{n-1} .
$$

Let $T \in \mathcal{T}_{n}$, let $k$ be the smallest integer such that $T^{k}(n)=0$, and let $j_{l}=T^{l}(n), l=$ $0, \cdots, k$ (so $j_{0}=n$ and $j_{k}=0$ ). Since $0=s_{n} \leq s_{j_{1}} \leq \cdots \leq s_{j_{k}}=t$, we have

$$
t=\sum_{l=0}^{k-1}\left(s_{j_{l+1}}-s_{j_{l}}\right) \leq \sum_{l=0}^{k-1}\left\langle s_{j_{l+1}}-s_{j_{l}}\right\rangle \leq k \prod_{l=0}^{k-1}\left\langle s_{j_{l+1}}-s_{j_{l}}\right\rangle
$$

and so

$$
t^{\rho} \prod_{l=0}^{k-1} G_{T\left(j_{l}\right)}\left(s_{T\left(j_{l}\right)}-s_{j_{l}}\right) \leq k^{\rho} \prod_{l=0}^{k-1}\left\langle s_{T\left(j_{l}\right)}-s_{j_{l}}\right\rangle^{\rho} G_{T\left(j_{l}\right)}\left(s_{T\left(j_{l}\right)}-s_{j_{l}}\right) .
$$

Hence, we can estimate

$$
\int_{0}^{\infty} t^{\rho}\left\|\left[V, \tau_{\lambda V}^{t}(A)\right]\right\| \mathrm{d} t \leq M_{A} \sum_{n=1}^{\infty} n^{\rho}|\lambda|^{n-1} I_{n}
$$

where

$$
I_{n}=\sum_{T \in \mathcal{T}_{n}} \bar{N}(T) \int_{0=s_{n} \leq s_{n-1} \cdots \leq s_{0}<\infty} \prod_{j=1}^{n}\left\langle s_{T(j)}-s_{j}\right\rangle^{\rho} G_{T(j)}\left(s_{T(j)}-s_{j}\right) \mathrm{d} s_{0} \cdots \mathrm{d} s_{n-1} .
$$

Applying the Botvich-Guta-Maassen integral estimate [BGM] in the same way as in Section 2.1 of [JOP] we deduce that

$$
\sup _{|\lambda| \leq \lambda_{V, \rho}} \sum_{n=1}^{\infty}|\lambda|^{n-1} I_{n}<\infty
$$

and the statement follows.

The next theorem establishes the rate of approach to the NESS and is of independent interest.

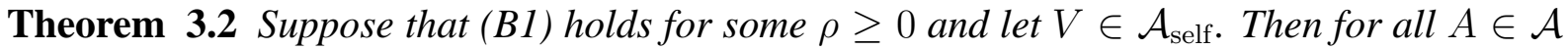
and $0<\Lambda<\lambda_{V, \rho}$,

$$
\sup _{|\lambda| \leq \Lambda}\left|\omega\left(\tau_{\lambda V}^{t}(A)\right)-\omega_{\lambda V}^{+}(A)\right|=O\left(\langle t\rangle^{-\rho}\right)
$$


Proof. The identity

$$
\tau_{0}^{-u} \circ \tau_{\lambda V}^{u}(A)-\tau_{0}^{-t} \circ \tau_{\lambda V}^{t}(A)=\mathrm{i} \lambda \int_{t}^{u} \tau_{0}^{-s}\left(\left[V, \tau_{\lambda V}^{s}(A)\right]\right) \mathrm{d} s
$$

and the $\tau_{0}$-invariance of $\omega$ yield that

$$
\omega_{\lambda V}^{+}(A)-\omega\left(\tau_{\lambda V}^{t}(A)\right)=\mathrm{i} \lambda \int_{t}^{\infty} \omega\left(\left[V, \tau_{\lambda V}^{s}(A)\right]\right) \mathrm{d} s .
$$

Hence,

$$
\sup _{|\lambda| \leq \Lambda}\left|\omega\left(\tau_{\lambda V}^{t}(A)\right)-\omega_{\lambda V}^{+}(A)\right| \leq \Lambda\langle t\rangle^{-\rho} \sup _{|\lambda| \leq \Lambda} \int_{t}^{\infty}\langle s\rangle^{\rho} \|\left[V, \tau_{\lambda V}^{s}(A)\right] \mid \mathrm{d} s,
$$

and the statement follows from Proposition 3.1.

Proof of Theorem 1.3. Let $\mathcal{V}$ be the set of all $V \in \mathcal{A}_{\text {self }}$ such that for some $0<|\lambda|<\lambda_{V}$, $\operatorname{Ep}\left(\omega_{\lambda V}^{+}\right)>0$. By Part (2) of Theorem 1.2 the function $\lambda \mapsto \operatorname{Ep}\left(\omega_{\lambda V}^{+}\right)$is analytic for $|\lambda|<\lambda_{V}$, and so for any $V \in \mathcal{V}$ the entropy production can vanish only for a discrete set of $\lambda$ 's in ]$-\lambda_{V}, \lambda_{V}[$.

To prove that $\mathcal{V}$ is dense in $\mathcal{A}_{\text {self }}$ we shall use Theorem 1.1. As we have already discussed, (B1)-(B2) imply that (A1)-(A3) hold, and we only need to verify that (A4) also holds.

For a given $V \in \mathcal{A}_{\text {self }}$, the observable

$$
A=\frac{1}{\lambda} \sigma_{\lambda V}
$$

does not depend on $\lambda$ and belongs to $\mathcal{A}$. Since $\rho>1$, Theorem 3.2 yields that for all $0<\Lambda<$ $\lambda_{V, \rho}$

$$
\sup _{|\lambda| \leq \Lambda, t \geq 0}\left|\int_{0}^{t}\left(\omega\left(\tau_{\lambda V}^{s}(A)\right)-\omega_{\lambda V}^{+}(A)\right) \mathrm{d} s\right|<\infty .
$$

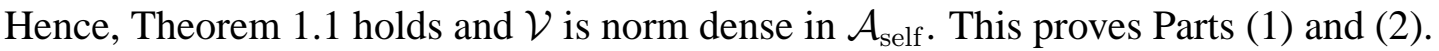

To prove (3), let $V, W \in \mathcal{A}_{\text {self. }}$ Set $\bar{n}=\max \left(\bar{n}_{V}, \bar{n}_{W}\right), K=K_{V}+K_{W}, \mathcal{F}=\mathcal{F}(V) \cup \mathcal{F}(W)$,

$$
\ell=\int_{0}^{\infty} \max _{f, g \in \mathcal{F}}\left|\left(f, \mathrm{e}^{\mathrm{i} t h_{0}} g\right)\right| \mathrm{d} t
$$

and

$$
\lambda_{V W}=\left(2 \bar{n} K(\max \{1,\|f\| \mid f \in \mathcal{F}\})^{2 \bar{n}-1} \ell\right)^{-1} \frac{(2 \bar{n}-1)^{2 \bar{n}-1}}{(2 \bar{n}-2)^{2 \bar{n}-2}}
$$

The proof of Theorem 1.1 in $[\mathrm{JOP}]$ gives that for all $A \in \mathcal{A}$,

$$
\sup _{|\lambda| \leq \lambda_{V W},|\epsilon| \leq 1} \int_{0}^{\infty}\left\|\left[V, \tau_{\lambda(V+\epsilon W)}^{t}(A)\right]\right\| \mathrm{d} t<\infty,
$$


and

$$
\lim _{\epsilon \rightarrow 0} \sup _{|\lambda| \leq \lambda_{V W}} \int_{0}^{\infty}\left\|\left[V, \tau_{\lambda(V+\epsilon W)}^{t}(A)-\tau_{\lambda V}^{t}(A)\right]\right\| \mathrm{d} t=0
$$

The estimate

$$
\begin{aligned}
\left|\omega_{\lambda(V+\epsilon W)}^{+}\left(\sigma_{\lambda(V+\epsilon W)}\right)-\omega_{\lambda V}^{+}\left(\sigma_{\lambda V}\right)\right| \leq|\lambda \epsilon|\left\|\delta_{\varsigma}(W)\right\|+\left|\omega_{\lambda(V+\epsilon W)}^{+}\left(\sigma_{\lambda V}\right)-\omega_{\lambda V}^{+}\left(\sigma_{\lambda V}\right)\right| \\
\leq|\lambda \epsilon|\left\|\delta_{\varsigma}(W)\right\|+|\lambda| \int_{0}^{\infty}\left\|\left[V, \tau_{\lambda(V+\epsilon W)}^{t}\left(\sigma_{\lambda V}\right)-\tau_{\lambda V}^{t}\left(\sigma_{\lambda V}\right)\right]\right\| \mathrm{d} t
\end{aligned}
$$

implies that if $\omega_{\lambda V}\left(\sigma_{\lambda V}\right)>0$ for some $0<|\lambda|<\lambda_{V W}$, then $\omega_{\lambda(V+\epsilon W)}^{+}\left(\sigma_{\lambda(V+\epsilon W)}\right)>0$ for $\epsilon$ small enough. Hence, if $V \in \mathcal{V}$ and $W \in \mathcal{A}_{\text {self }}$, then for $\epsilon$ small enough, $V+\epsilon W \in \mathcal{V}$.

\section{References}

[AM] Aizenstadt, V.V., Malyshev, V.A.: Spin interaction with an ideal Fermi gas. J. Stat. Phys. 48, 51 (1987).

[AJPP] Aschbacher, W., Jakšić, V., Pautrat, Y., Pillet, C.-A.: Topics in non-equilibrium quantum statistical mechanics. In Open Quantum Systems III. S. Attal, A. Joye, C.-A. Pillet editors. Lecture Notes in Mathematics 1882, Springer, New York (2006).

[AS] Aschbacher, W., Spohn, H.: A remark on the strict positivity of entropy production. Lett. Math. Phys. 75, 17 (2006).

[BGM] Botvich, D.D., Guta, M., Maassen, H.: Stability of Bose dynamical systems and branching theory. Preprint (mp_arc 99-130).

[BM1] Botvich, D.D., Malyshev, V.A.: Unitary equivalence of temperature dynamics for ideal and locally perturbed Fermi gas. Commun. Math. Phys. 91, 301 (1983).

[BM2] Botvich, D.D., Malyshev, V.A.: Asymptotic completeness and all that for an infinite number of fermions. In Many-Particle Hamiltonians: Spectra and Scattering. Minlos, R. A. editor. Advances in Soviet Mathematics 5, 39, AMS, Providence (1991).

[BKR] Bratteli, O., Kishimoto, A., Robinson, D.W.: Stability properties and the KMS condition. Commun. Math. Phys. 61, 209 (1978).

[BR1] Bratteli, O., Robinson, D. W.: Operator Algebras and Quantum Statistical Mechanics 1. Springer-Verlag, Berlin (1987).

[BR2] Bratteli, O., Robinson, D. W.: Operator Algebras and Quantum Statistical Mechanics 2. Second edition, Springer-Verlag, Berlin (1996).

[FMU] Fröhlich, J., Merkli, M., Ueltschi, D.: Dissipative transport: thermal contacts and tunneling junctions. Ann. Henri Poincaré 4, 897 (2004). 
[JOP] Jakšić, V., Ogata, Y., Pillet, C.-A.: The Green-Kubo formula for locally interacting Fermionic open system. To appear in Ann. Henri Poincaré.

[JP1] Jakšić, V., Pillet, C.-A.: On a model for quantum friction III: Ergodic properties of the spinboson system. Commun. Math. Phys. 178, 627 (1996).

[JP2] Jakšić, V., Pillet, C-A.: On entropy production in quantum statistical mechanics. Commun. Math. Phys. 217, 285 (2001).

[JP3] Jakšić, V., Pillet, C.-A.: Mathematical theory of non-equilibrium quantum statistical mechanics. J. Stat. Phys. 108, 787 (2002).

[JP4] Jakšić, V., Pillet, C.-A.: In preparation.

[OP] Ohya, M., Petz, D.: Quantum Entropy and its Use. Springer-Verlag, Berlin (1993).

[P] Pillet, C.-A.: Quantum dynamical systems. In Open Quantum Systems I. S. Attal, A. Joye, C.-A. Pillet editors. Lecture Notes in Mathematics 1880, Springer, New York (2006).

[Ro] Robinson, D.W.: Return to equilibrium. Commun. Math. Phys. 31, 171 (1973).

[Ru1] Ruelle, D.: Natural nonequilibrium states in quantum statistical mechanics. J. Stat. Phys. 98, 57 (2000).

[Ru2] Ruelle, D.: Entropy production in quantum spin systems. Commun. Math. Phys. 224, 3 (2001). 